\title{
Novel Results for Asymmetrically Coupled Fractional Neurons
}

\author{
Carla M. A. Pinto \\ School of Engineering, Polytechnic of Porto, Center for Mathematics of the \\ University of Porto, Rua Dr António Bernardino de Almeida, 431, 4200-072 \\ Porto, Portugal, e-mail: cap@isep.ipp.pt
}

\begin{abstract}
We consider a fractional-order model of two asymmetrically coupled spiking neurons. The dynamical behavior of the two neurons is modeled by the fractional-order Hodgkin-Huxley equations. Simulations of the model for distinct values of the order of the fractional derivative, $\alpha$, and of the coupling constants, $k_{1}, k_{2}$, show interesting features, such as relaxation oscillations, mixed-mode oscillations, small oscillations, and localized solutions. Moreover, a adds extra complexity to the dynamics of the model. These differences may explain certain differences in processing similar tasks in the human brain.
\end{abstract}

Keywords: asymmetric; coupled; neurons; fractional Hodgkin-Huxley equations

\section{Introduction}

In 1952, Hodgkin and Huxley [1] conducted experiments, in the squid axon, aimed at a better understanding of the mechanisms and rules governing the flow of the electric current in a nerve cell, during an action potential. The derived equations, known as the Hodgkin-Huxley $(\mathrm{HH})$ equations, have had a decisive influence on the understanding of the neuronal function since then [2], [3], [4], [5]. Phenomena such as in-phase synchronization, anti-phase synchronization, bursting, localization, small oscillations, mixed-mode oscillations, have been modeled by the $\mathrm{HH}$ equations.

Synchronization is observed in specific areas of the brain in patients suffering from epilepsy and Parkinson's disease [6]. On the other hand, tasks such as processing sensory information, only occur in synchronized neurons. Localized solutions in oscillatory systems are associated with a partition of the oscillators in two distinct sets. One set is described by oscillators with high amplitudes and the other by oscillators with small amplitudes [7]. These types of patterns may be good approximations for the dynamics of working memory, in a biologically reasonable parameter region [8]. Relaxation oscillations are solutions defined by 
long periods of quasi-static behavior interspersed with short periods of rapid transition. They are analyzed in the context of the canard phenomenon [9]. A solution showing a combination of traits of relaxation oscillations and small oscillations is defined as a mixed mode oscillation. The later may also be generated by the canard mechanism.

In [2] the authors simulate an integer-order asymmetrically coupled system of two $\mathrm{HH}$ equations. They find localized solutions, small oscillations, relaxation oscillations, and mixed-mode oscillations, for certain values of the coupling constants. Localized solutions are seen for negative values of the two coupling constants and when the ratio $\frac{k_{1}}{k_{2}}$ between the two constants is far from to -1 . Relaxation oscillations occur when this ratio is close to -1 , and mixed mode oscillations are the states in between.

Keeping the aforementioned ideas in mind, in this paper, we propose a fractional order model for the dynamics of two asymmetrically coupled $\mathrm{HH}$ equations, for variation of the order of the fractional derivative and various temperatures. We consider that the coupling is diffusive and is only done in the voltage term. This is, to our best knowledge, not been the issue of any previous research. In Section 2, we introduce the FO model of asymmetrically coupled HH equations. In Section 3 we show and discuss the outcomes of the simulations of the model. In the last section, we conclude our work.

\subsection{Non-Integer Order Differentiation}

Non-integer order, aka fractional order (FO), differentiation and integration are generalizations of the well-known differentiation and integration of integer order. FO systems have been widely applied, for a couple of decades now, to solve problems in engineering, biology, physics, to name a few [10, 11, 12, 13, 14].

There are several definitions of FO derivatives. The most commonly used are the Caputo, the Grünwald-Letnikov, and the Riemann-Liouville derivatives [10]. The GL derivative is given by the equation (1).

${ }_{a}^{G L} D_{t}^{\alpha} f(t)=\lim _{h \rightarrow 0} \frac{1}{h^{\alpha}} \sum_{0}^{\left[\frac{t-a}{h}\right]}(-1)^{k}\left(\begin{array}{l}\alpha \\ k\end{array}\right) f(t-k h), t>a, \alpha>0$

In 2015, Caputo and Fabrizio (CF) [15] proposed a new definition for the fractional order derivative. The update with respect to previous definitions is the new non-singular kernel operator. This novel derivative has been used in groundwater and thermal problems. Moreover, Atangana et al. apply the CF derivative to find the solutions of the Fisher's reaction-diffusion equation and of the Baggs-Freedman model [16, 17]. 


\section{The Fractional-Order Asymmetrically Coupled System of Two HH Equations}

The Hodgkin-Huxley equations (1) are a system of $4 \times 4$ ordinary differential equations (ODEs). They were derived by Hodgkin and Huxley in 1952 [1] to model the electrical behavior of the squid axon. The first equation refers to the trans-membrane potential dynamics, $v(t)$, for a single neuron, in response to an external stimulus $I$, and as a function of the ion currents. The ion currents are mostly three, one for the sodium $\left(\mathrm{Na}^{+}\right)$, one for the potassium $\left(\mathrm{K}^{+}\right)$, and one for a leakage current, associated with other ions, where calcium is included. The ions' conductance's are described by the other three equations of the model.

$\frac{c_{m} d v}{d t}=f(v, m, n, h)-I$

$\frac{d m}{d t}=\Phi\left(\alpha_{m}(v)(1-m)-\beta_{m}(v) m\right)$

$\frac{d n}{d t}=\Phi\left(\alpha_{n}(v)(1-n)-\beta_{n}(v) n\right)$

$\frac{d h}{d t}=\Phi\left(\alpha_{h}(v)(1-h)-\beta_{h}(v) h\right)$

where $C_{m}$ is the membrane capacitance, $\Phi=3^{\frac{T-6.3}{10}}$, is the temperature compensating factor. The function $f$ is defined as:

$f(v, m, n, h)=-g_{L}\left(v-V_{L}\right)-g_{N a} m^{3} h\left(v-V_{N a}\right)-g_{K} n^{4}\left(v-V_{k}\right)$

The functions $\alpha_{i}(v)$ and $\beta_{i}(v), i=m, n, h$, are given in [1] as:

$\alpha_{m}(v)=\psi\left(\frac{v+25}{10}\right), \alpha_{n}(v)=0.1 \psi\left(\frac{v+10}{10}\right), \alpha_{h}(v)=0.07 \psi\left(\frac{v}{20}\right)$

$\beta_{m}(v)=4 e^{\left(\frac{v}{18}\right)}, \beta_{n}(v)=\frac{1}{8} e^{\left(\frac{v}{80}\right)}, \beta_{h}(v)=\left(1+e^{\left(\frac{v+30}{10}\right)}\right)^{(-1)}$

and $\psi=\left\{\begin{array}{cc}\frac{x}{e^{x}-1}, & x \neq 0 \\ 1, & x=0\end{array}\right.$.

The asymmetrically coupled system of two fractional-order HH equations is thus given by:

$\frac{c_{m} d^{\alpha} v_{1}}{d t^{\alpha}}=f\left(v_{1}, m_{1}, n_{1}, h_{1}\right)-I-k_{1}\left(v_{1}-v_{2}\right)$

$\frac{d m_{1}}{d t}=\Phi\left(\alpha_{m_{1}}\left(v_{1}\right)\left(1-m_{1}\right)-\beta_{m_{1}}\left(v_{1}\right) m_{1}\right)$

$\frac{d n_{1}}{d t}=\Phi\left(\alpha_{n_{1}}\left(v_{1}\right)\left(1-n_{1}\right)-\beta_{n_{1}}\left(v_{1}\right) n_{1}\right)$

$\frac{d h_{1}}{d t}=\Phi\left(\alpha_{h_{1}}\left(v_{1}\right)\left(1-h_{1}\right)-\beta_{h_{1}}\left(v_{1}\right) h_{1}\right)$

$\frac{c_{m} d^{\alpha} v_{2}}{d t^{\alpha}}=f\left(v_{2}, m_{2}, n_{2}, h_{2}\right)-I-k_{2}\left(v_{2}-v_{1}\right)$ 


$$
\begin{aligned}
& \frac{d m_{2}}{d t}=\Phi\left(\alpha_{m_{2}}\left(v_{2}\right)\left(1-m_{2}\right)-\beta_{m_{2}}\left(v_{2}\right) m_{2}\right) \\
& \frac{d n_{2}}{d t}=\Phi\left(\alpha_{n_{2}}\left(v_{2}\right)\left(1-n_{2}\right)-\beta_{n_{2}}\left(v_{2}\right) n_{2}\right) \\
& \frac{d h_{2}}{d t}=\Phi\left(\alpha_{h_{2}}\left(v_{2}\right)\left(1-h_{2}\right)-\beta_{h_{2}}\left(v_{2}\right) h_{2}\right)
\end{aligned}
$$

where $k_{1}, k_{2}$ are the coupling constants, and $\alpha$ is the order of the fractional derivative.

\section{Numerical Simulations}

In this section we show simulations of the FO asymmetrically coupled $\mathrm{HH}$ equations model (4), for several values of the order of the fractional derivative, $\alpha$, and distinct coupling constants, $k_{1}, k_{2}$. In Table 1 , we list the values of the $\mathrm{HH}$ parameters fixed in the simulations. In Table 2, can be found the initial conditions and the values for the varied parameters. The symmetrically coupled FO HH equations model is studied in [18].

Table 1

Values of the Hodgkin-Huxley parameters used in the simulations

\begin{tabular}{|ccc|}
\hline Parameters & Values & Units \\
$C_{m}$ & 1.0 & $\mu \mathrm{F} / \mathrm{cm}^{2}$ \\
$T$ & $6.3,16.0,26.0$ & ${ }^{o} \mathrm{C}$ \\
$V_{N a}$ & -115.0 & $\mathrm{mV}$ \\
$V_{K}$ & 12.0 & $\mathrm{mV}$ \\
$V_{L}$ & -10.599 & $\mathrm{mV}$ \\
$g_{N a}$ & 120.0 & $\mathrm{mS} / \mathrm{cm}^{2}$ \\
$g_{K}$ & 36 & $\mathrm{mS} / \mathrm{cm}^{2}$ \\
$g_{L}$ & 0.3 & $\mathrm{mS} / \mathrm{cm}^{2}$ \\
\hline
\end{tabular}

Table 2

Initial conditions and parameter values used in the simulations

\begin{tabular}{|llc|c|}
\hline Fig. & \multicolumn{1}{c|}{ Initial conditions } & \multicolumn{1}{c|}{$\boldsymbol{T}, \boldsymbol{I}$} & $\boldsymbol{k}_{\mathbf{1}}, \boldsymbol{k}_{\mathbf{2}}$ \\
\hline $1,2,3$ & $(-22.18,0.43,0.65,0.07$, & $26^{\circ} \mathrm{C}, 155$ & $-0.2,-2.01$ \\
& $-20.81,0.38,0.63,0.08)$ & & \\
$4,5,6$ & $(-10.67,0.17,0.56,0.17$, & $16^{\circ} C, 60$ & $0.7,-1.1$ \\
& $-18.91,0.67,0.74,0.05)$ & & \\
$7,8,9$ & $(-16.21,0.36,0.60,0.10$, & $16^{\circ} C, 60$ & $0.5,-1.1$ \\
& $1.39,0.04,0.52,0.28)$ & & \\
$10,11,12$ & $(-26.01,0.48,0.64,0.07$, & $20^{\circ} C, 155$ & $-0.1,-2.0$ \\
& $-7.43,0.12,0.63,0.10)$ & & \\
\hline
\end{tabular}


Figures 1-3 depict small oscillations of the FO coupled system (3), for $T=26.0, I=155$, and $\alpha=1.0,0.8,0.4$, respectively. One can observe a decrease in the amplitude of the periodic orbits with the order of the fractional derivative, $\alpha$, and a slight increase in the spiking frequency. Faster transients are observed for smaller $\alpha$. In Figures 4-6, we show relaxation oscillations of the model (3), for $T=16.0, I=60$, and $\alpha=1.0,0.8,0.4$, respectively. As $\alpha$ is decreased the spiking frequency of the two neurons increases. In Figures 7-8, we show mixedmode oscillations of the model (2), for $T=16.0, I=60$, and $\alpha=1.0,0.8$, respectively. Fixing all other parameters and decreasing only $\alpha$ to 0.4 , the mixedmode oscillations are lost, and a relaxation oscillation appears, see Figure 9. Thus, $\alpha$, causes a, non-expected, change in the behavior of the system (3). Localized solutions are shown in Figures $10-12$, for $T=20.0, I=155$, and $\alpha=$ $1.0,0.8,0.4$, respectively. Moreover, we note a decrease in the amplitude and an increase in the spiking frequency of the neurons as $\alpha$ decreases from 1 .

As it can be observed from the numerical simulations, the asymmetrically coupled FO model of two HH equations has a rich dynamical behavior. Mixed-mode oscillations, relaxation oscillations, small oscillations, and localized solutions, are common in specific regions of the coupling constants. These regions agree with the results of paper [2], for most values of $\alpha$. This means that localized solutions are seen for negative values of the two coupling constants and when the ratio $\frac{k_{1}}{k_{2}}$ between the two constants is far from to -1 . Relaxation oscillations occur when this ratio is close to -1 , and mixed mode oscillations are the states in between. Nevertheless, the value of the fractional order derivative, $\alpha$, adds extra complexity to the behavior of the model. We saw in Figure 9 an 'expected' mixed-mode oscillation tend towards a relaxation oscillation, when all other parameters 'suggested' a mixed-mode oscillation. This 'complexity' may be associated with differences in the human brain, when processing and storing information $[8,7]$, when responding to certain stimuli, amongst others. Further study is needed in order to infer the importance of the order of the fractional derivative in these models of spiking neurons. 


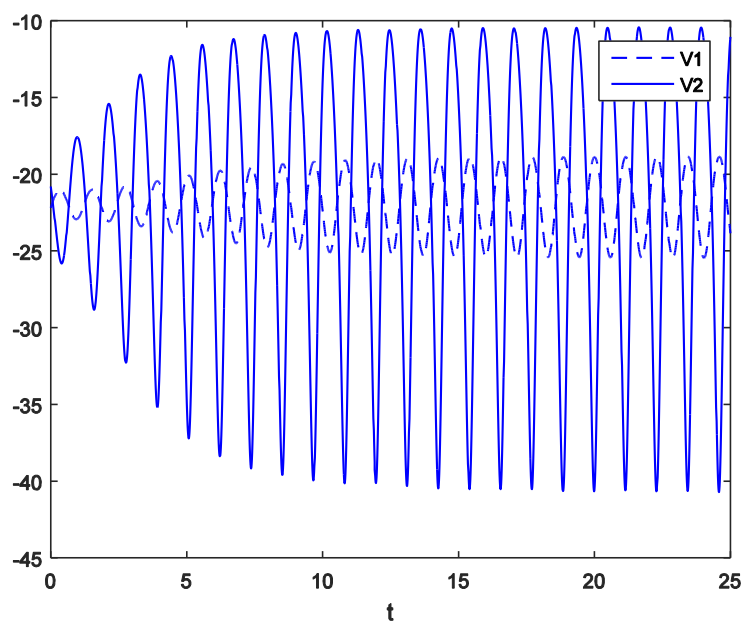

Figure 1

Small oscillations of the FO model (2) for $T=26.0, I=155$, and $\alpha=1.0$

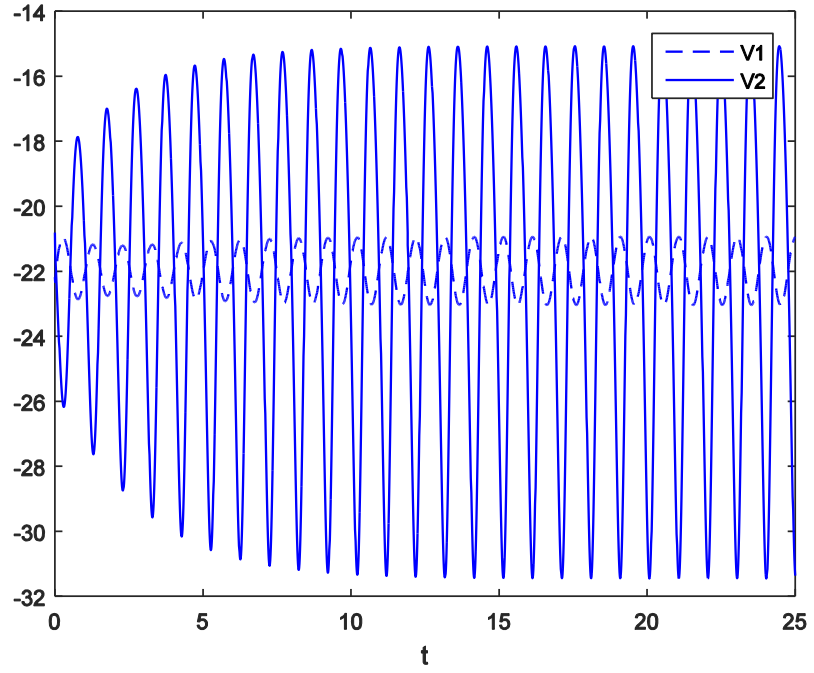

Figure 2

Small oscillations of the FO model (2) for $T=26.0, I=155$ and $\alpha=0.8$ 


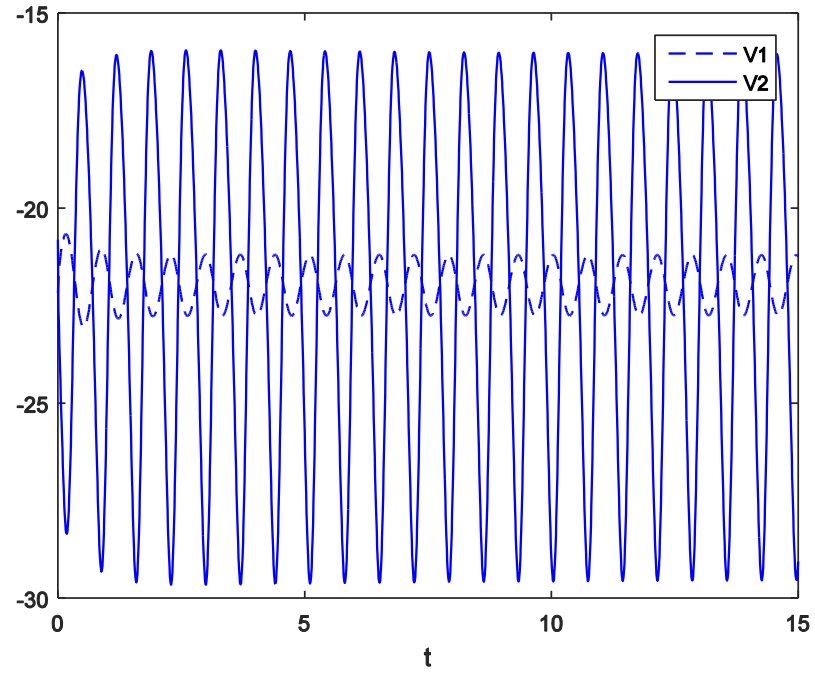

Figure 3

Small oscillations of the FO model (2) for $T=26.0, I=155$, and $\alpha=0.4$

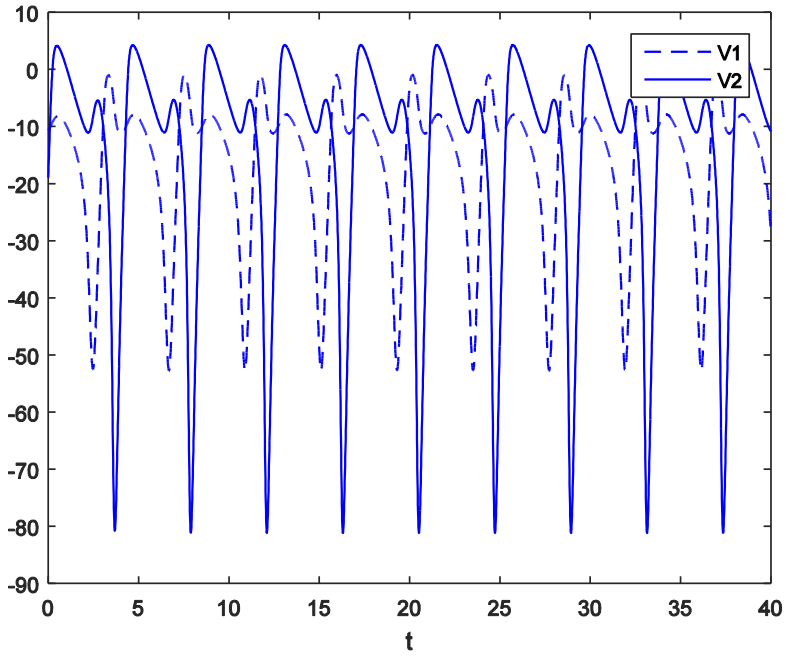

Figure 4

Relaxation oscillations of the FO model (2) for $T=16.0, I=60$, and $\alpha=1.0$ 


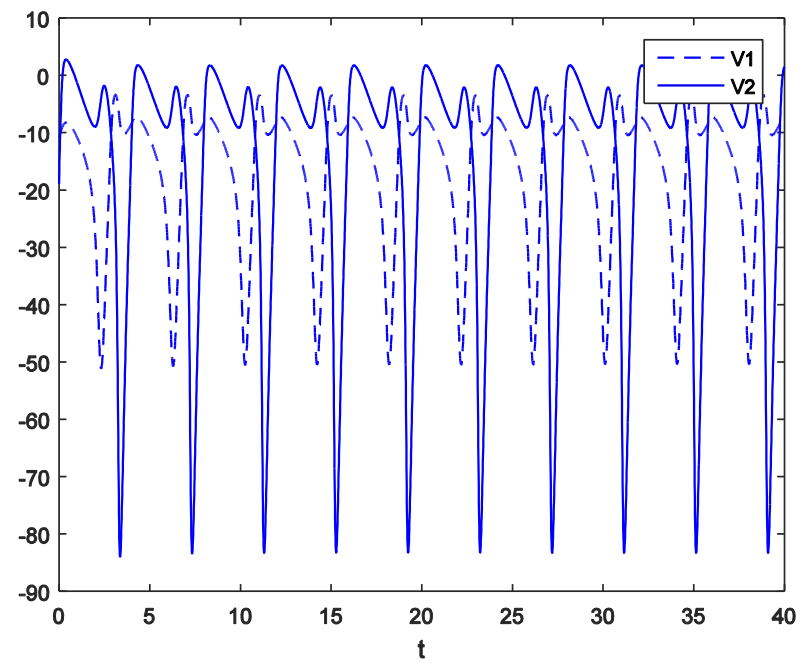

Figure 5

Relaxation oscillations of the FO model (2) for $T=16.0, I=60$, and $\alpha=0.8$

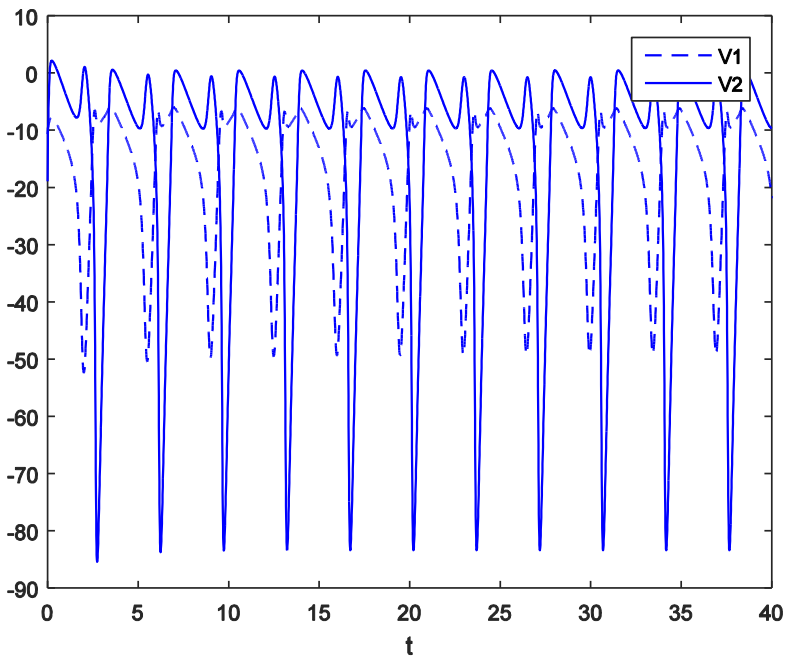

Figure 6

Relaxation oscillations of the FO model (2) for $T=16.0, I=60$, and $\alpha=0.4$ 


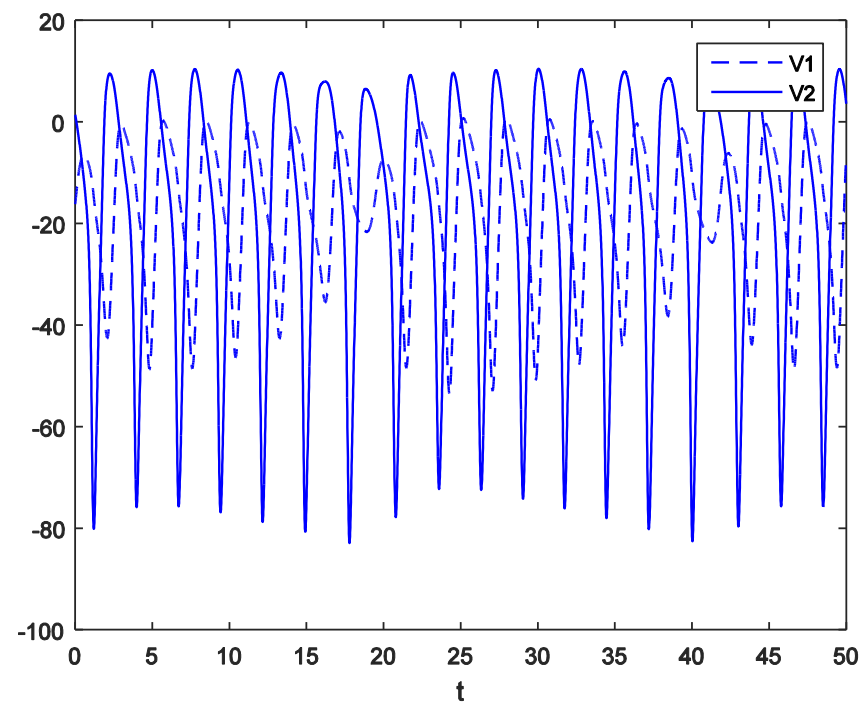

Figure 7

Mixed-mode oscillations of the FO model (2) for $T=16.0, I=60$, and $\alpha=1.0$

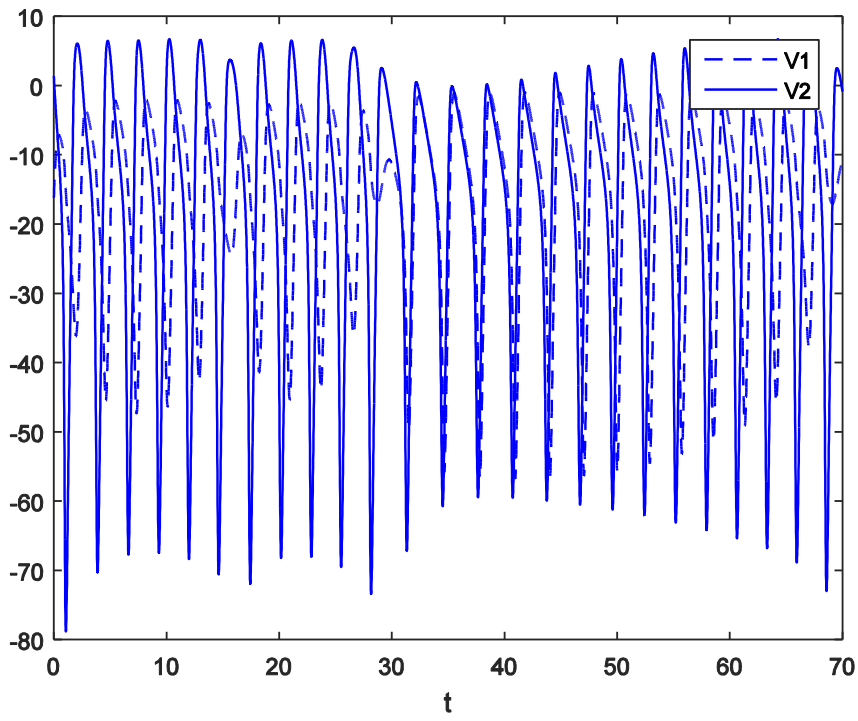

Figure 8

Mixed-mode oscillations of the FO model (2) for $T=16.0, I=60$, and $\alpha=0.8$ 


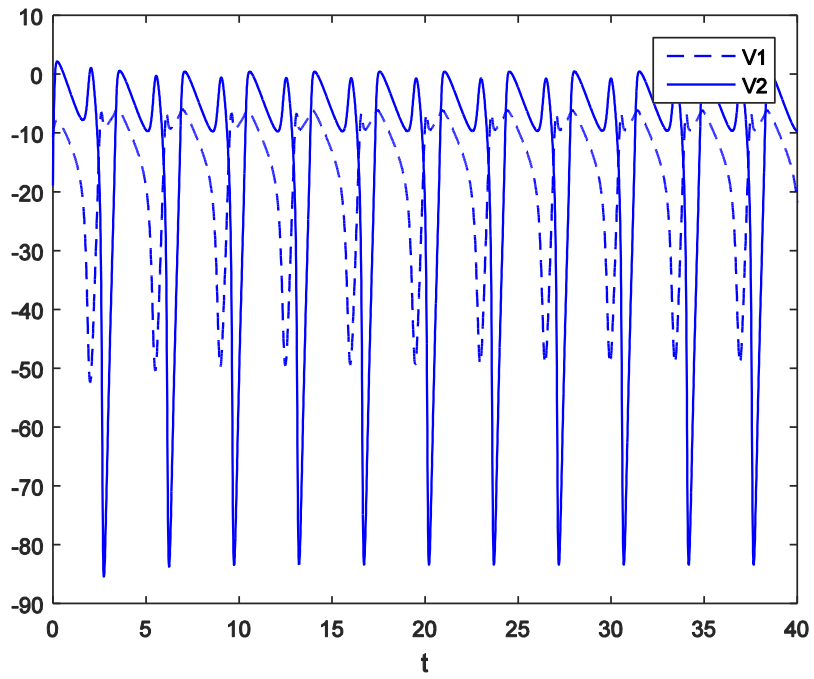

Figure 9

Relaxation oscillations of the FO model (2) for $T=16.0, I=60$, and $\alpha=0.4$

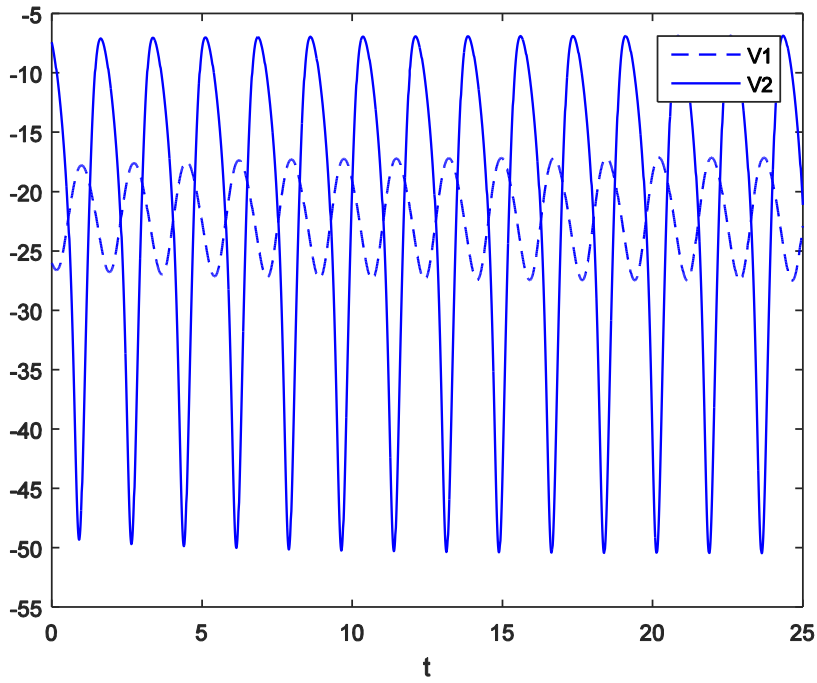

Figure 10

Localized solutions of the FO model (2) for $\mathrm{T}=20.0, I=155$, and $\alpha=1.0$ 


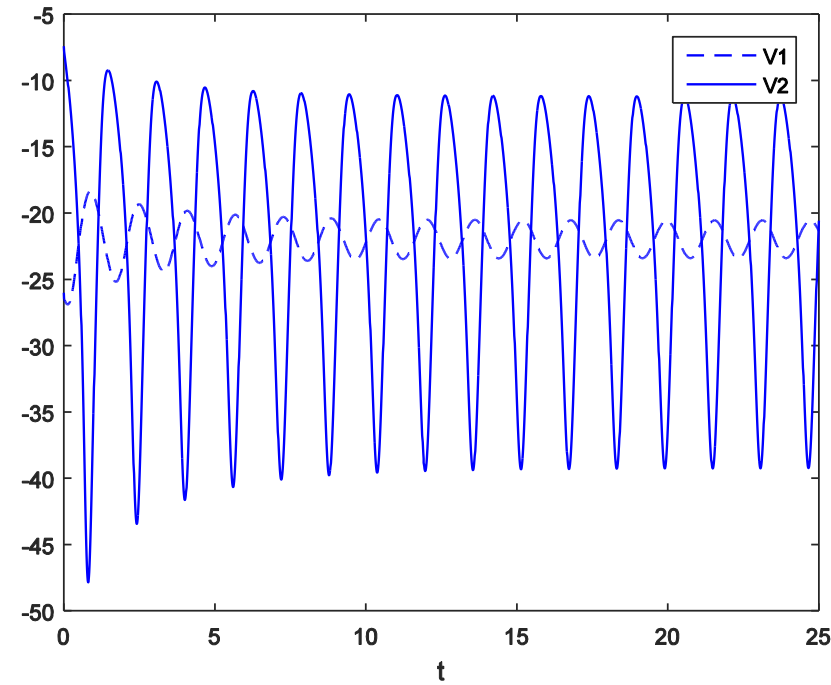

Figure 11

Localized solutions of the FO model (2) for $\mathrm{T}=20.0, I=155$, and $\alpha=0.8$

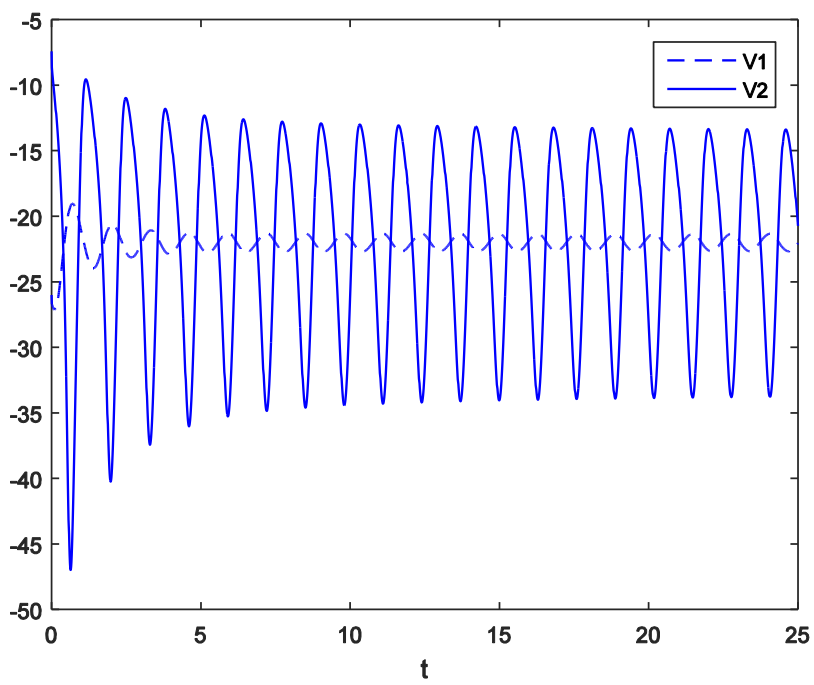

Figure 12

Localized solutions of the FO model (2) for $\mathrm{T}=20.0, I=155$, and $\alpha=0.4$ 


\section{Conclusions}

Herein we observed an asymmetrically coupled model of two FO HH equations. The model is rich in terms of diversity of dynamic patterns. One can distinguish mixed-mode oscillations, small oscillations, relaxation oscillations and localized solutions for certain parameters (coupling constants) regions. Moreover, the value of the order of the fractional derivative comprises more complexity to the coupled FO model. This may be used to explain differences in the human brain when storing and processing memories or when reacting to the same stimuli. More work is needed in order to fully understand the vast diversity of patterns in the model.

\section{Acknowledgement}

The author was partially supported by CMUP (UID/MAT/00144/2013), which is funded by FCT (Portugal) with national (MEC) and European structural funds (FEDER), under the partnership agreement PT2020 and by PAPRE-Programa de Apoio à Publicação em Revistas Científicas de Elevada Qualidade from Polytechnic Institute of Porto.

\section{References}

[1] Hodgkin, A. L., Huxley, A. F., A Quantitative Description of Membrane Current and Its Application to Conduction and Excitation in Nerve. In $J$ Physiol (Lond), Vol. 117, 1952, pp. 500-544

[2] M. Krupa and C. M. A. Pinto, Curious Phenomena in Two Coupled Neurons. In Proceedings of the $26^{\text {th }}$ IASTED International Conference on Modelling, Identification and Control, 2007, pp. 387-390

[3] I. S. Labouriau and C. M. Alves-Pinto, Loss of Synchronization in Partially Coupled Hodgkin-Huxley Equations. In Bulletin of Mathematical Biology, Vol. 66, 2004, pp. 539-557

[4] H. H. Sherief, A. M. A. El-Sayed, S. H. Behiry, W. E. Raslan, Using Fractional Derivatives to Generalize the Hodgkin-Huxley Model. In: Fractional Dynamics and Control, New York, NY: Springer New York, 2012, pp. 275-282

[5] A. M. Nagy and N. H. Sweilam, An Efficient Method for Solving Fractional Hodgkin-Huxley Model. In Physics Letters A, Vol. 378, 2014, pp. 1980-1984

[6] J. Modolo, B. Bhattacharya, R. Edwards, J. Campagnaud, A. Legros, A. Beuter, Using a Virtual Cortical Module Implementing a Neural Field Model to Modulate Brain Rhythms in Parkinson's Disease. In Frontiers of Neuroscience, Vol. 4, Article 45, 2010, p. 11

[7] H. G. Rotstein, N. Kopell, A. M. Zhabotinsky, I. R. Epstein, A Canard Mechanism for Localization in Systems of Globally Coupled Oscillators. In: SIAM J. Appl. Math, Vol. 63(6), 2003, pp. 1998-2019 
[8] C. P. Fall, T. J. Lewis, J. Rinzel, Background-Activity-Dependent Properties of a Network Model for Working Memory that Incorporates Cellular Bistability, In: Biol. Cybern., Vol. 93(2), 2005, pp. 109-118

[9] Krupa M, Szmolyan P. Relaxation Oscillations and Canard Explosion. In: $J$ Diff Equations, Vol. 174, 2001, pp. 312-368

[10] A. A. Kilbas and H. M. Srivastava and J. J. Trujillo. Theory and Applications of Fractional Differential Equations 204, North-Holland Mathematics Studies, Elsevier, Amsterdam, 2006

[11] F. Mainardi, Fractional Relaxation-Oscillation and Fractional DiffusionWave Phenomena. In Chaos Solitons \& Fractals, Vol. 7, 1996, pp. 14611477

[12] D. Baleanu, S. I. Muslih, Lagrangian Formulation of Classical Fields within Riemann-Liouville Fractional Derivatives In Phys. Scr., Vol. 72(2-3), 2005, 119-121

[13] R. De Keyser, C. I. Muresan, C. M. Ionescu, A Novel Auto-Tuning Method for Fractional Order PI/PD Controllers. In ISA Transactions, Vol. 62, 2016, pp. 268-275

[14] C. M. A. Pinto, A. R. M. Carvalho, New Findings on the Dynamics of HIV and TB Coinfection Models. In Appl. Math. Comput., Vol. 242, 2014 36-46

[15] M. Caputo, M. Fabrizio, A New Definition of Fractional Derivative without Singular Kernel, In Progr. Fract. Differ. Appl., Vol. 2, 2015, 73-85

[16] A. Atangana, On the New Fractional Derivative and Application to Nonlinear Fisher's Reaction-Diffusion Equation. In Appl. Math. Comput., Vol. 273, 2016, 948-956

[17] A. Atangana, I. Koca, On the New Fractional Derivative and Application to Nonlinear Baggs and Freedman Model. In J. Nonlinear Sci. Appl., Vol. 9, 2016, 2467-2480

[18] C. M. A. Pinto, Coupled Fractional Spiking Neurons. In: Proceedings of 2016 IEEE International Conference on Systems, Man, and Cybernetics, February 2017, 000487-000491 